Kragujevac Journal of Mathematics

Volume 42(2) (2018), Pages 161-175.

\title{
CERTAIN GEOMETRIC PROPERTIES OF ANALYTIC FUNCTIONS ASSOCIATED WITH THE CONVOLUTIONS
}

\author{
POONAM SHARMA ${ }^{1}$ AND ADITYA K. BAJPAI ${ }^{2}$ \\ (Dedicated to the Memory of Academician Petru Tudor Mocanu)
}

(1931-2016)

\begin{abstract}
In this paper, we define convolutions $(f * g([\alpha]))(z)$ and $(f * h([\alpha]))(z)$ of functions analytic in the open unit disk with some non-zero parameter $\alpha$, satisfying certain recurring relations. Making use of admissible function method introduced by Miller and Mocanu, certain geometric properties of these convolutions are obtained. Taking specific forms of the functions $g([\alpha])$ and $h([\alpha])$, some consequences of our results are also given.
\end{abstract}

\section{INTRODUCTION}

Let $\mathcal{H}$ denote the class of functions analytic in the open unit $\mathbb{U}=z:|z|<1$, and for $k \in \mathbb{N}=\{1,2, \ldots\}$ and $a \in \mathbb{C}$, let

$$
\mathcal{H}[a, k]=\left\{f \in \mathcal{H}: f(z)=a+a_{k} z^{k}+a_{k+1} z^{k+1}+\cdots\right\} .
$$

Also, let $\mathcal{A}$ denote a subclass of functions in $\mathcal{H}[0, k]$ which are of the form

$$
f(z)=z+\sum_{n=2}^{\infty} a_{n} z^{n} .
$$

Let $g([\alpha]) \in \mathcal{A}$ be of the form

$$
g([\alpha])(z)=z+\sum_{n=2}^{\infty} b_{n}([\alpha]) z^{n}
$$

Key words and phrases. analytic functions, convolution, Dziok-Srivastava convolution operator, Jung-Kim-Srivastava integral operator, multiplier operator.

2010 Mathematics Subject Classification. Primary: 30C45, 30C50.

Received: April 19, 2016.

Accepted: December 20, 2016. 
and $h([\alpha]) \in \mathcal{A}$ be of the form

$$
h([\alpha])(z)=z+\sum_{n=2}^{\infty} c_{n}([\alpha]) z^{n},
$$

with some bounded coefficients $b_{n}([\alpha])$ and $c_{n}([\alpha])$ depending on a non-zero parameter $\alpha \in \mathbb{C}$, satisfying for some $0 \neq \lambda_{\alpha}, \kappa_{\alpha} \in \mathbb{C}$, the recurring relations:

$$
\lambda_{\alpha} g([\alpha+1])(z)=\left(\lambda_{\alpha}-1\right) g([\alpha])(z)+z(g([\alpha]))^{\prime}(z)
$$

and

$$
\kappa_{\alpha} h([\alpha-1])(z)=\left(\kappa_{\alpha}-1\right) h([\alpha])(z)+z(h([\alpha]))^{\prime}(z) .
$$

A convolution (Hadamard product) $*$ of $f \in \mathcal{A}$ of the form (1.1) and $g \in \mathcal{A}$ of the form

$$
g(z)=z+\sum_{n=2}^{\infty} b_{n} z^{n}
$$

is defined by

$$
f(z) * g(z)=(f * g)(z)=z+\sum_{n=2}^{\infty} a_{n} b_{n} z^{n} .
$$

Involving convolution defined by (1.6), it is easily verified from (1.4) and (1.5) that for some non-zero complex constants $\lambda_{\alpha}$ and $\kappa_{\alpha}$,

$$
\lambda_{\alpha}(f * g([\alpha+1]))(z)=\left(\lambda_{\alpha}-1\right)(f * g([\alpha]))(z)+z\left(f * g([\alpha])^{\prime}(z)\right.
$$

and

$$
\kappa_{\alpha}(f * h([\alpha-1]))(z)=\left(\kappa_{\alpha}-1\right)(f * h([\alpha]))(z)+z(f * h([\alpha]))^{\prime}(z) .
$$

In the Geometric Function Theory various linear operators have been defined so far, out of which few well known are the Dziok-Srivastava convolution operator [6], a linear operator associated with a generalized Bessel function studied in [1], the Srivastava-Attiya linear operator [21], the Jung-Kim-Srivastava integral operator [7], a multiplier operator introduced in [13] (see also [14,18]) and a new fractional operator studied in [17]. These linear operators include several operators cited therein, and are defined as follows.

The Dzoik-Srivastava operator $[6],{ }_{p} H_{q}\left(\left[\alpha_{1}\right]\right): \mathcal{A} \rightarrow \mathcal{A}$, is defined by

$$
{ }_{p} H_{q}\left(\left[\alpha_{1}\right]\right) f(z)=z_{p} F_{q}\left(\alpha_{1}, \ldots, \alpha_{p} ; \beta_{1}, \ldots, \beta_{q} ; z\right) * f(z),
$$

where

$$
{ }_{p} F_{q}\left(\alpha_{1}, \ldots, \alpha_{p} ; \beta_{1}, \ldots, \beta_{q} ; z\right)=\sum_{n=0}^{\infty} \frac{\prod_{i=1}^{p}\left(\alpha_{i}\right)_{n}}{\prod_{i=1}^{q}\left(\beta_{i}\right)_{n}} \frac{z^{n}}{n !}
$$


$p \leq q+1, p, q \in \mathbb{N}_{0}=\{0,1,2, \ldots\}, \alpha_{i}, \beta_{i} \in \mathbb{C}\left(\beta_{i} \neq 0,-1,-2, \ldots\right), z \in \mathbb{U}$, is the generalized hypergeometric function $\left(\left[12\right.\right.$, p. 19]). The symbol $(\lambda)_{n}$ is the Pochhammer symbol defined by

$$
(\lambda)_{n}=\frac{\Gamma(\lambda+n)}{\Gamma(\lambda)}=\lambda(\lambda+1)(\lambda+2) \cdots(\lambda+n-1), \quad n \in \mathbb{N},(\lambda)_{0}=1 .
$$

Recently, in [1], a linear operator $B_{k}^{c}: \mathcal{A} \rightarrow \mathcal{A}$ is defined in terms of a generalized Bessel function $\omega_{p, b, c}(z)[2,3]$ of the first kind of order $p$, by

$$
B_{k}^{c} f(z)=\varphi_{k, c}(z) * f(z)
$$

where

$$
\begin{aligned}
\varphi_{k, c}(z) & :=\varphi_{p, b, c}(z)=2^{p} \Gamma\left(p+\frac{b+1}{2}\right) z^{1-p / 2} \omega_{p, b, c}(\sqrt{z}) \\
& =z+\sum_{n=2}^{\infty} \frac{(-c)^{n-1}}{4^{n-1}(k)_{n-1}(n-1) !} z^{n}
\end{aligned}
$$

$k=p+\frac{b+1}{2} \in \mathbb{C}(\neq 0,-1,-2, \ldots), c, b, p \in \mathbb{C}, z \in \mathbb{C}$.

The Srivastava-Attiya linear operator $[21], J_{a, b}: \mathcal{A} \rightarrow \mathcal{A}$, is defined in terms of generalized Hurwitz-Lerch Zeta function $\phi(b, a, z)[22]$ by

$$
J_{a, b} f(z)=G_{a, b}(z) * f(z),
$$

where

$$
G_{a, b}(z)=(b+1)^{a}\left(\phi(b, a, z)-b^{-a}\right)=z+\sum_{n=2}^{\infty}\left(\frac{b+1}{b+n}\right)^{a} z^{n},
$$

$b \in \mathbb{C}(b \neq 0,-1,-2, \ldots), a \in \mathbb{C}, z \in \mathbb{U}$.

The Jung-Kim-Srivastava integral operator [7], $Q_{\beta}^{\alpha}: \mathcal{A} \rightarrow \mathcal{A}$, satisfy

$$
z\left(Q_{\beta}^{\alpha} f(z)\right)^{\prime}=(\alpha+\beta) Q_{\beta}^{\alpha-1} f(z)+(1-\alpha-\beta) Q_{\beta}^{\alpha} f(z),
$$

where

$$
Q_{\beta}^{\alpha} f(z)=\left(\begin{array}{c}
\alpha+\beta \\
\beta
\end{array}\right)^{\alpha-1} t^{\beta-1} f(t) \mathrm{d} t, \quad \alpha>0, \beta>-1 .
$$

The multiplier operator, $\Im_{k, \mu}^{m}: \mathcal{A} \rightarrow \mathcal{A}$, recently studied in [13] (see also [14], [18]) is defined for $m \in \mathbb{Z}=\{\ldots,-2,-1,0,1,2, \ldots\}, \mu>-1, k>0$, by

$$
\left\{\begin{array}{lll}
\Im_{k, \mu}^{m} f(z)=f(z), & & m=0, \\
\Im_{k, \mu}^{m} f(z)=\frac{\mu+1}{k} z^{1-\frac{\mu+1}{k}} \int_{0}^{z} t^{\frac{\mu+1}{k}-2} \Im_{k, \mu}^{m+1} f(t) \mathrm{d} t, & & m \in \mathbb{Z}^{-}=\{-1,-2, \ldots\}, \\
\Im_{k, \mu}^{m} f(z)=\frac{k}{\mu+1} z^{2-\frac{\mu+1}{k}} \frac{\mathrm{d}}{\mathrm{d} t}\left(z^{\frac{\mu+1}{k}-1} \Im_{k, \mu}^{m-1} f(z)\right), & & m \in \mathbb{Z}^{+}=\{1,2, \ldots\} .
\end{array}\right.
$$

A new fractional operator $\mathbb{D}_{\lambda}^{\nu, n}: \mathcal{A} \rightarrow \mathcal{A}$ is defined in [17] as a composition of the Ruscheweyh operator $\mathcal{R}^{\nu}[15]$, the Sălăgean operator $\mathcal{D}^{n}[16]$ and a fractional 
differintegral operator $\Omega_{z}^{\lambda}[10]$ by

$$
\mathbb{D}_{\lambda}^{\nu, n} f(z)=\mathcal{R}^{\nu} \mathcal{D}^{n} \Omega_{z}^{\lambda} f(z)
$$

The series expansion of $\mathbb{D}_{\lambda}^{\nu, n} f(z)$ for $f \in \mathcal{A}$ of the form (1.1) is given by

$$
\mathbb{D}_{\lambda}^{\nu, n} f(z)=z+\sum_{k=1}^{\infty} \frac{(\nu+1)_{k}}{(2-\lambda)_{k}}(k+1)^{n+1} a_{k+1} z^{k+1},
$$

where $-\infty<\lambda<2, \nu>-1, n \in \mathbb{N}_{0}, z \in \mathbb{U}$. In this paper, making use of the admissible function method introduced by Miller and Mocanu [8, Theorem 2.3b, p. $28]$, certain geometric properties of the convolutions $f * g([\alpha])$ and $f * h([\alpha])$ of functions in the class $\mathcal{A}$, are investigated, where the functions $g([\alpha])$ and $h([\alpha])$ are of the form (1.2) and (1.3), respectively. Some consequences of our main results taking some specific forms of the functions $g([\alpha])(z)$ and $h([\alpha])(z)$ are also considered. A result of Miller and Mocanu [8, Theorem 4.6a, p. 244] giving a sufficiency condition for the function $f$ to be starlike in $\mathbb{U}$ follows from our main results.

Note that certain other geometric properties of these convolutions are studied by the authors in $[19,20]$. Further, involving certain linear operators, some more results based on geometric properties of the analytic functions using the admissible function method, may be found in the recent work $[1,17]$.

\section{Main Results}

To prove our main results, we first define the following class of admissible functions which is the special case of [8, Definition 2.3a, p. 27].

Definition 2.1. [8, Case 2, p. 34] Let $k \geq 1, a \in \mathbb{C}$ with Re $a>0$. An admissible function $\psi: \mathbb{C}^{3} \times \mathbb{U} \rightarrow \mathbb{C}$ is said to be in the class $\Phi_{k}[a]$, if $\psi$ satisfies the admissibility condition:

$$
\operatorname{Re} \psi(\rho i, \sigma, \mu+i v ; z) \leq 0, \quad z \in \mathbb{U}
$$

where $\rho, \sigma, \mu, v \in \mathbb{R}$,

$$
\sigma \leq-\frac{k}{2} \frac{|a-i \rho|^{2}}{\operatorname{Re} a}, \quad \sigma+\mu \leq 0
$$

For the admissible functions of the class $\Phi_{k}[a]$, we give following lemma which is special cases of the result [8, Theorem $2.3 \mathrm{~b}$, p. 28].

Lemma 2.1. [8, Theorem 2.3 (i), (ii), p. 35] Let $\varphi \in \Phi_{k}[a]$. If $p \in \mathcal{H}[a, k]$, then

$$
\operatorname{Re} \varphi\left(p(z), z p^{\prime}(z), z^{2} p^{\prime \prime}(z) ; z\right)>0 \Rightarrow \operatorname{Re} p(z)>0 .
$$

We use Lemma 2.1 to obtain our main results. Our first result gives a geometric property of the convolution $f * g([\alpha])$ which is contained in the following theorem.

Theorem 2.1. Let $g([\alpha+j]) \in \mathcal{A}$ be defined by (1.2) and for $\lambda_{\alpha+j} \neq 0, j=0,1$, let $\Omega: \mathbb{C}^{3} \rightarrow \mathbb{C}$ be an admissible function such that

$$
\operatorname{Re} \Omega\left(\rho i, \rho i+\frac{\sigma}{\lambda_{\alpha}},\left(\frac{1}{\lambda_{\alpha}}+\frac{1}{\lambda_{\alpha+1}}\right) \sigma+\frac{\sigma+\mu}{\lambda_{\alpha} \lambda_{\alpha+1}}+\left(\rho+\frac{v}{\lambda_{\alpha} \lambda_{\alpha+1}}\right) i\right) \leq 0,
$$


where $\rho, \sigma, \eta, v \in \mathbb{R}$, such that

$$
\sigma \leq-\frac{1}{2}\left(1+\rho^{2}\right), \quad \mu \leq \frac{1}{2}\left(1+\rho^{2}\right) .
$$

If $f \in \mathcal{A}$ satisfies the condition:

$$
\operatorname{Re} \Omega\left(\frac{(f * g([\alpha]))(z)}{z}, \frac{(f * g([\alpha+1]))(z)}{z}, \frac{(f * g([\alpha+2]))(z)}{z}\right)>0, \quad z \in \mathbb{U},
$$

then

$$
\operatorname{Re}\left(\frac{(f * g([\alpha]))(z)}{z}\right)>0, \quad z \in \mathbb{U}
$$

Proof. Let the function $w(z)$ be defined by

$$
w(z)=\frac{(f * g([\alpha]))(z)}{z},
$$

then it follows that $w \in \mathcal{H}[1,1]$. On differentiating (2.2) logarithmically, we obtain

$$
\frac{w^{\prime}(z)}{w(z)}=\frac{(f * g([\alpha]))^{\prime}(z)}{(f * g([\alpha]))(z)}-\frac{1}{z},
$$

from which it follows with the help of (2.2) that

$$
(f * g([\alpha]))^{\prime}(z)=w(z)+z w^{\prime}(z) .
$$

Now, from (1.7), we have the identity

$$
\frac{(f * g([\alpha+1]))(z)}{z}=\left(1-\frac{1}{\lambda_{\alpha}}\right) \frac{(f * g([\alpha]))(z)}{z}+\frac{1}{\lambda_{\alpha}}(f * g([\alpha]))^{\prime}(z),
$$

which on using (2.2) and (2.3) yields

$$
\begin{aligned}
\frac{(f * g([\alpha+1]))(z)}{z} & =\left(1-\frac{1}{\lambda_{\alpha}}\right) w(z)+\frac{1}{\lambda_{\alpha}}\left(w(z)+z w^{\prime}(z)\right) \\
& =w(z)+\frac{1}{\lambda_{\alpha}} z w^{\prime}(z) .
\end{aligned}
$$

On replacing $\alpha$ by $\alpha+1$ in identity (2.4), we obtain

$$
\frac{(f * g([\alpha+2]))(z)}{z}=\left(1-\frac{1}{\lambda_{\alpha+1}}\right) \frac{(f * g([\alpha+1]))(z)}{z}+\frac{1}{\lambda_{\alpha+1}}(f * g([\alpha+1]))^{\prime}(z) \text {. }
$$

Again on performing elementary calculations with the use of the (2.5) and (2.6), we get

$$
\begin{aligned}
\frac{(f * g([\alpha+2]))(z)}{z}= & w(z)+\left(\frac{1}{\lambda_{\alpha}}+\frac{1}{\lambda_{\alpha+1}}+\frac{1}{\lambda_{\alpha} \lambda_{\alpha+1}}\right) z w^{\prime}(z) \\
& +\frac{1}{\lambda_{\alpha} \lambda_{\alpha+1}} z^{2} w^{\prime \prime}(z) .
\end{aligned}
$$


On putting

$$
w(z)=r, \quad z w^{\prime}(z)=s, \quad z^{2} w^{\prime \prime}(z)=t,
$$

and using a transformation from $\mathbb{C}^{3}$ to $\mathbb{C}^{3}$ :

$$
\left\{\begin{array}{l}
k_{1}=r \\
k_{2}=r+\frac{s}{\lambda_{\alpha}} \\
k_{3}=r+\left(\frac{1}{\lambda_{\alpha}}+\frac{1}{\lambda_{\alpha+1}}+\frac{1}{\lambda_{\alpha} \lambda_{\alpha+1}}\right) s+\frac{t}{\lambda_{\alpha} \lambda_{\alpha+1}}
\end{array}\right.
$$

we get

$$
\Omega\left(k_{1}, k_{2}, k_{3}\right)=\varphi(r, s, t) .
$$

If we put $r=\rho i, s=\sigma$ and $t=\mu+i v$ in the above transformation, we obtain (in view of the hypothesis) that $\varphi \in \Phi_{1}[1]$, and we have

$$
k_{3}=\left(\frac{1}{\lambda_{\alpha}}+\frac{1}{\lambda_{\alpha+1}}\right) \sigma+\frac{\sigma+\mu}{\lambda_{\alpha} \lambda_{\alpha+1}}+\left(\rho+\frac{v}{\lambda_{\alpha} \lambda_{\alpha+1}}\right) i
$$

where

$$
\sigma \leq-\frac{1}{2}\left(1+\rho^{2}\right) \text { and } \mu \leq \frac{1}{2}\left(1+\rho^{2}\right) \Rightarrow \sigma+\mu \leq 0 .
$$

Thus, on applying Lemma 2.1, the condition:

$$
\begin{aligned}
& \operatorname{Re} \Omega\left(\frac{(f * g([\alpha]))(z)}{z}, \frac{(f * g([\alpha+1]))(z)}{z}, \frac{(f * g([\alpha+2]))(z)}{z}\right) \\
= & \operatorname{Re} \varphi\left(w(z), z w^{\prime}(z), z^{2} w^{\prime \prime}(z)\right)>0, \quad z \in \mathbb{U},
\end{aligned}
$$

implies that

$$
\operatorname{Re} w(z)=\operatorname{Re}\left(\frac{(f * g([\alpha]))(z)}{z}\right)>0, \quad z \in \mathbb{U},
$$

which proves the result (2.1) of Theorem 2.1.

Following similar lines of the proof of Theorem 2.1, on using the identity (1.8), we may easily get following result which gives a geometric property of the convolution $f * h([\alpha])$.

Theorem 2.2. Let $h([\alpha-j]) \in \mathcal{A}$ be defined by (1.3) and for $\kappa_{\alpha-j} \neq 0, j=0,1$ let $\Pi: \mathbb{C}^{3} \rightarrow \mathbb{C}$ be an admissible function such that

$$
\operatorname{Re} \Pi\left(\rho i, \rho i+\frac{\sigma}{\kappa_{\alpha}},\left(\frac{1}{\kappa_{\alpha}}+\frac{1}{\kappa_{\alpha-1}}\right) \sigma+\frac{\sigma+\mu}{\kappa_{\alpha} \kappa_{\alpha-1}}+\left(\rho+\frac{v}{\kappa_{\alpha} \kappa_{\alpha-1}}\right) i\right) \leq 0,
$$

where $\rho, \sigma, \eta, v \in \mathbb{R}$, such that

$$
\sigma \leq-\frac{1}{2}\left(1+\rho^{2}\right), \quad \mu \leq \frac{1}{2}\left(1+\rho^{2}\right) .
$$

If $f \in \mathcal{A}$ satisfies the condition:

$$
\operatorname{Re} \Pi\left(\frac{(f * h([\alpha]))(z)}{z}, \frac{(f * h([\alpha-1]))(z)}{z}, \frac{(f * h([\alpha-2]))(z)}{z}\right)>0, \quad z \in \mathbb{U}
$$


then

$$
\operatorname{Re}\left(\frac{(f * h([\alpha]))(z)}{z}\right)>0, \quad z \in \mathbb{U}
$$

Let

$$
p(z)=\frac{1}{\lambda_{\alpha+1}} \log \frac{(f * g([\alpha+1]))(z)}{z},
$$

with $\frac{(f * g([\alpha+1]))(z)}{z} \neq 0$ for $z \neq 0$ and $\frac{(f * g([\alpha+1]))(z)}{z}=1$ for $z=0$. Then $p(z)$ is analytic in $\mathbb{U}$ with $\stackrel{z}{p}(0)=0$. Then with the use of identity (1.7), we obtain

$$
p^{\prime}(z)=\frac{1}{\lambda_{\alpha+1}}\left(\frac{(f * g([\alpha+1]))^{\prime}(z)}{(f * g([\alpha+1]))(z)}-\frac{1}{z}\right) .
$$

Again on performing elementary calculations with the use of the (2.5), we get

$$
p^{\prime}(z)=\frac{1}{z}\left(\frac{(f * g([\alpha+2]))(z)}{(f * g([\alpha+1]))(z)}-1\right) .
$$

Now we define

$$
\{f * g[\alpha], z\}=\left[\frac{1}{z}\left(\frac{(f * g([\alpha+2]))(z)}{(f * g([\alpha+1]))(z)}-1\right)\right]^{\prime}-\frac{1}{2}\left[\frac{1}{z}\left(\frac{(f * g([\alpha+2]))(z)}{(f * g([\alpha+1]))(z)}-1\right)\right]^{2} \text {. }
$$

Our next result is as follows.

Theorem 2.3. Let $g([\alpha+j]) \in \mathcal{A}$ be defined by (1.2) and for reals $\lambda_{\alpha+j} \neq 0, j=0,1$, let $\digamma: \mathbb{C}^{3} \rightarrow \mathbb{C}$ be an admissible function such that

$$
\operatorname{Re} \digamma\left(\rho i, \frac{1}{\lambda_{\alpha+1}}\left[\lambda_{\alpha+1}-\lambda_{\alpha}+\tau i\right], \frac{1}{\lambda_{\alpha+1}}[\xi-\eta i]\right) \leq 0,
$$

where $\rho, \tau, \xi, \eta, \mu \in \mathbb{R}$,

$$
\lambda_{\alpha} \rho^{2}-\rho \tau \leq-\frac{1}{2}\left(1+\rho^{2}\right) \text { and } \mu \leq \frac{1}{2}\left(1+\rho^{2}\right) .
$$

If $f \in \mathcal{A}$ with $(f * g[\alpha+j])(z) \neq 0$ in $\mathbb{U}$ for $j=0,1$, satisfies the condition:

$$
\operatorname{Re} \digamma\left(\frac{(f * g[\alpha+1])(z)}{(f * g[\alpha])(z)}, \frac{(f * g[\alpha+2])(z)}{(f * g[\alpha+1])(z)}, z^{2}\{f * g[\alpha], z\}\right)>0, \quad z \in \mathbb{U},
$$

then

$$
\operatorname{Re}\left(\frac{(f * g[\alpha+1])(z)}{(f * g[\alpha])(z)}\right)>0, \quad z \in \mathbb{U}
$$

Proof. Let the function $q(z)$ be defined by

$$
q(z)=\frac{(f * g[\alpha+1])(z)}{(f * g[\alpha])(z)}
$$


then it follows that $q \in \mathcal{H}[1,1]$. On performing elementary calculations with the use of the identity (1.7), we get

$$
\frac{1}{z}\left(\frac{f * g([\alpha+2])(z)}{f * g([\alpha+1])(z)}-1\right)=\frac{1}{\lambda_{\alpha+1}}\left[\frac{q^{\prime}(z)}{q(z)}+\lambda_{\alpha} \frac{q(z)-1}{z}\right] .
$$

Hence, from (2.10), we get

$$
\frac{f * g([\alpha+2])(z)}{f * g([\alpha+1])(z)}=\frac{1}{\lambda_{\alpha+1}}\left[\lambda_{\alpha} q(z)+\frac{z q^{\prime}(z)}{q(z)}+\lambda_{\alpha+1}-\lambda_{\alpha}\right] .
$$

Again, on differentiating (2.10), we get

$$
\begin{aligned}
& {\left[\frac{1}{z}\left(\frac{(f * g([\alpha+2]))(z)}{(f * g([\alpha+1]))(z)}-1\right)\right]^{\prime} } \\
= & \frac{1}{\lambda_{\alpha+1}}\left\{\frac{q^{\prime \prime}(z)}{q(z)}-\left(\frac{q^{\prime}(z)}{q(z)}\right)^{2}+\lambda_{\alpha} \frac{z q^{\prime}(z)-q(z)+1}{z^{2}}\right\},
\end{aligned}
$$

and squaring (2.10),

$$
\begin{aligned}
& {\left[\frac{1}{z}\left(\frac{(f * g([\alpha+2]))(z)}{(f * g([\alpha+1]))(z)}-1\right)\right]^{2} } \\
= & \frac{1}{\lambda_{\alpha+1}^{2}}\left[\left(\frac{q^{\prime}(z)}{q(z)}\right)^{2}+\frac{\lambda_{\alpha}^{2}}{z^{2}}\left(q^{2}(z)-2 q(z)+1\right)+\frac{2 \lambda_{\alpha}}{z^{2}}\left(z q^{\prime}(z)-\frac{z q^{\prime}(z)}{q(z)}\right)\right] .
\end{aligned}
$$

Substituting from (2.11) and (2.12) in (2.7), we get

$$
\begin{aligned}
z^{2}\{f * g[\alpha], z\}= & \frac{1}{\lambda_{\alpha+1}}\left\{\frac{z^{2} q^{\prime \prime}(z)}{q(z)}-\left(1+\frac{1}{2 \lambda_{\alpha+1}}\right)\left(\frac{z q^{\prime}(z)}{q(z)}\right)^{2}+\frac{\lambda_{\alpha}}{\lambda_{\alpha+1}} \frac{z q^{\prime}(z)}{q(z)}\right. \\
& +\lambda_{\alpha}\left(1-\frac{1}{\lambda_{\alpha+1}}\right) z q^{\prime}(z)-\lambda_{\alpha}\left(1-\frac{\lambda_{\alpha}}{\lambda_{\alpha+1}}\right) q(z) \\
& \left.-\frac{\lambda_{\alpha}^{2}}{2 \lambda_{\alpha+1}} q^{2}(z)+\lambda_{\alpha}\left(1-\frac{\lambda_{\alpha}}{2 \lambda_{\alpha+1}}\right)\right\} .
\end{aligned}
$$

On putting

$$
q(z)=r, \quad z q^{\prime}(z)=s, \quad z^{2} q^{\prime \prime}(z)=t,
$$

and using a transformation from $\mathbb{C}^{3}$ to $\mathbb{C}^{3}$ :

we get

$$
\left\{\begin{aligned}
l_{1}= & r \\
l_{2}= & \frac{1}{\lambda_{\alpha+1}}\left[\lambda_{\alpha} r+\frac{s}{r}+\lambda_{\alpha+1}-\lambda_{\alpha}\right], \\
l_{3}= & \frac{1}{\lambda_{\alpha+1}}\left[\frac{t}{r}-\left(1+\frac{1}{2 \lambda_{\alpha+1}}\right)\left(\frac{s}{r}\right)^{2}+\frac{\lambda_{\alpha}}{\lambda_{\alpha+1}} \frac{s}{r}+\lambda_{\alpha}\left(1-\frac{1}{\lambda_{\alpha+1}}\right) s\right. \\
& \left.-\lambda_{\alpha}\left(1-\frac{\lambda_{\alpha}}{\lambda_{\alpha+1}}\right) r-\frac{\lambda_{\alpha}^{2}}{2 \lambda_{\alpha+1}} r^{2}+\lambda_{\alpha}\left(1-\frac{\lambda_{\alpha}}{2 \lambda_{\alpha+1}}\right)\right],
\end{aligned}\right.
$$

$$
\digamma\left(l_{1}, l_{2}, l_{3}\right)=\varphi(r, s, t) .
$$


If we put $r=\rho i, s=\sigma$ and $t=\mu+i v$ in the above transformation, we obtain (in view of the hypothesis) that $\varphi \in \Phi_{1}[1]$, and we have

$$
\begin{aligned}
l_{1}= & \rho i, \\
l_{2}= & \frac{1}{\lambda_{\alpha+1}}\left[\left(\lambda_{\alpha} \rho-\frac{\sigma}{\rho}\right) i+\lambda_{\alpha+1}-\lambda_{\alpha}\right] \\
= & \frac{1}{\lambda_{\alpha+1}}\left[\lambda_{\alpha+1}-\lambda_{\alpha}+\tau i\right], \\
l_{3}= & \frac{1}{\lambda_{\alpha+1}}\left[\frac{\nu}{\rho}+\left(1+\frac{1}{2 \lambda_{\alpha+1}}\right)\left(\frac{\sigma}{\rho}\right)^{2}+\frac{\lambda_{\alpha}^{2}}{2 \lambda_{\alpha+1}} \rho^{2}+\lambda_{\alpha}\left(1-\frac{1}{\lambda_{\alpha+1}}\right) \sigma\right. \\
& \left.+\lambda_{\alpha}\left(1-\frac{\lambda_{\alpha}}{2 \lambda_{\alpha+1}}\right)-\left\{\frac{\mu}{\rho}+\frac{\lambda_{\alpha}}{\lambda_{\alpha+1}} \frac{\sigma}{\rho}+\lambda_{\alpha}\left(1-\frac{\lambda_{\alpha}}{\lambda_{\alpha+1}}\right) \rho\right\} i\right] \\
= & \frac{1}{\lambda_{\alpha+1}}[\xi-\eta i],
\end{aligned}
$$

where

$$
\begin{aligned}
\xi & =\frac{\nu}{\rho}+\left(1+\frac{1}{2 \lambda_{\alpha+1}}\right)\left(\frac{\sigma}{\rho}\right)^{2}+\frac{\lambda_{\alpha}^{2}}{2 \lambda_{\alpha+1}} \rho^{2}+\lambda_{\alpha}\left(1-\frac{\lambda_{\alpha}}{2 \lambda_{\alpha+1}}\right)+\lambda_{\alpha}\left(1-\frac{1}{\lambda_{\alpha+1}}\right) \sigma \\
\eta & =\left(\frac{\mu}{\rho}+\frac{\lambda_{\alpha}}{\lambda_{\alpha+1}} \frac{\sigma}{\rho}+\lambda_{\alpha}\left(1-\frac{\lambda_{\alpha}}{\lambda_{\alpha+1}}\right) \rho\right),
\end{aligned}
$$

are real and

$$
\sigma=\lambda_{\alpha} \rho^{2}-\rho \tau \leq-\frac{1}{2}\left(1+\rho^{2}\right) \wedge \mu \leq \frac{1}{2}\left(1+\rho^{2}\right) \Rightarrow \sigma+\mu \leq 0 .
$$

Thus, by Lemma 2.1, the condition:

$$
\begin{aligned}
& \operatorname{Re} \digamma\left(\frac{(f * g[\alpha+1])(z)}{(f * g[\alpha])(z)}, \frac{(f * g[\alpha+2])(z)}{(f * g[\alpha+1])(z)}, z^{2}\{f * g[\alpha], z\}\right) \\
= & \operatorname{Re} \varphi\left(q(z), z q^{\prime}(z), z^{2} q^{\prime \prime}(z)\right)>0, \quad z \in \mathbb{U},
\end{aligned}
$$

implies from (2.9) that

$$
\operatorname{Re} q(z)=\operatorname{Re}\left(\frac{(f * g[\alpha+1])(z)}{(f * g[\alpha])(z)}\right)>0, \quad z \in \mathbb{U},
$$

which proves the result (2.8) of Theorem 2.3.

Further, on using the identity (1.8), similar to the proof of Theorem 2.3, we may get following result.

Theorem 2.4. Let $h([\alpha-j]) \in \mathcal{A}$ be defined by (1.3) and for reals $\kappa_{\alpha-j} \neq 0, j=0,1$, let $\Lambda: \mathbb{C}^{3} \rightarrow \mathbb{C}$ be an admissible function such that

$$
\operatorname{Re} \Lambda\left(\rho i, \frac{1}{\kappa_{\alpha-1}}\left[\kappa_{\alpha-1}-\kappa_{\alpha}+\tau i\right], \frac{1}{\kappa_{\alpha-1}}[\xi-\eta i]\right) \leq 0
$$


where $\rho, \tau, \xi, \eta, \mu \in \mathbb{R}$,

$$
\kappa_{\alpha} \rho^{2}-\rho \tau \leq-\frac{1}{2}\left(1+\rho^{2}\right) \text { and } \mu \leq \frac{1}{2}\left(1+\rho^{2}\right) .
$$

If $f \in \mathcal{A}$ with $(f * h[\alpha-j])(z) \neq 0$ in $\mathbb{U}$ for $j=0,1$, satisfies the condition:

$$
\operatorname{Re} \Lambda\left(\frac{(f * h[\alpha-1])(z)}{(f * h[\alpha])(z)}, \frac{(f * h[\alpha-2])(z)}{(f * h[\alpha-1])(z)}, z^{2} \quad\{f * h[\alpha], z\}\right)>0, \quad z \in \mathbb{U},
$$

then

$$
\operatorname{Re}\left(\frac{(f * h[\alpha-1])(z)}{(f * h[\alpha])(z)}\right)>0, \quad z \in \mathbb{U} .
$$

Remark 2.1. Choosing $g([\alpha])(z)=\frac{z}{1-z}, z \in \mathbb{U}$, and $\lambda_{\alpha+j}=1$, for $j=0,1$, in (1.4) and choosing $h([\alpha])(z)=\frac{z}{1-z}, z \in \mathbb{U}$, and $\kappa_{\alpha-j}=1$, for $j=0,1$, in (1.5), we observe from (1.7) and (1.8) that

$$
\begin{gathered}
(f * g[\alpha])(z)=f(z)=(f * h[\alpha])(z), \\
(f * g[\alpha+1])(z)=z f^{\prime}(z)=(f * h[\alpha-1])(z), \\
(f * g[\alpha+2])(z)=z\left(z f^{\prime}(z)\right)^{\prime}=(f * h[\alpha-2])(z),
\end{gathered}
$$

and hence, in Theorem 2.3 or $2.4, z^{2}\{f * g[\alpha], z\}$ or $z^{2}\{f * h[\alpha], z\}$ reduces to the Schwarzian derivative of $f$ and the result of Theorem 2.3 or 2.4 coincides with the result proved by Miller and Mocanu [8, Theorem 4.6a, p. 244] giving a sufficiency condition for the function $f$ to be starlike in $\mathbb{U}$.

\section{Consequent Results}

In this section, we obtain some consequences of Theorems 2.1 and 2.2 by taking specific values of $\lambda_{\alpha}$ and $\kappa_{\alpha}$ by considering some specific forms of the functions $g([\alpha])(z)$ and $h([\alpha])(z)$.

On using recurring relation given in (1.7) for $\alpha=\alpha_{1}(\neq 0,-1)$ and on choosing $(f * g([\alpha+j]))(z)={ }_{p} H_{q}\left(\left[\alpha_{1}+j\right]\right) f(z)$ with $\lambda_{\alpha+j}=\alpha_{1}+j, j=0,1$, we get following result as a consequence of Theorem 2.1.

Corollary 3.1. Let ${ }_{p} H_{q}\left(\left[\alpha_{1}+j\right]\right)$ be defined by (1.9) and for $\alpha_{1}+j \neq 0, j=0,1$, let $\Omega_{1}: \mathbb{C}^{3} \rightarrow \mathbb{C}$ be an admissible function such that

$$
\operatorname{Re} \Omega_{1}\left(\rho i, \rho i+\frac{\sigma}{\alpha_{1}},\left(\frac{1}{\alpha_{1}}+\frac{1}{\alpha_{1}+1}\right) \sigma+\frac{\sigma+\mu}{\alpha_{1}\left(\alpha_{1}+1\right)}+\left(\rho+\frac{v}{\alpha_{1}\left(\alpha_{1}+1\right)}\right) i\right) \leq 0,
$$

where $\rho, \sigma, \eta, v \in \mathbb{R}$,

$$
\sigma \leq-\frac{1}{2}\left(1+\rho^{2}\right), \quad \mu \leq \frac{1}{2}\left(1+\rho^{2}\right) .
$$

If $f \in \mathcal{A}$ satisfies the condition:

$$
\operatorname{Re} \Omega_{1}\left(\frac{{ }_{p} H_{q}\left(\left[\alpha_{1}\right]\right) f(z)}{z}, \frac{{ }_{p} H_{q}\left(\left[\alpha_{1}+1\right]\right) f(z)}{z}, \frac{{ }_{p} H_{q}\left(\left[\alpha_{1}+2\right]\right) f(z)}{z}\right)>0, \quad z \in \mathbb{U},
$$


then

$$
\operatorname{Re}\left(\frac{{ }_{p} H_{q}\left(\left[\alpha_{1}\right]\right) f(z)}{z}\right)>0, \quad z \in \mathbb{U} .
$$

Choosing $\alpha=k+1(k \neq 1,0,-1, \ldots)$ and taking

$$
(f * h([\alpha-j]))(z)=B_{k+1-j}^{c} f(z)
$$

and $\kappa_{\alpha-j}=k-j, j=0,1$, we obtain from Theorem 2.2, the next result using the identity:

$$
k B_{k}^{c} f(z)=(k-1) B_{k+1}^{c} f(z)+z\left(B_{k+1}^{c} f(z)\right)^{\prime}(z) .
$$

Corollary 3.2. Let for $k \neq 1,0,-1, \ldots, B_{k}^{c} f(z)$ be defined by (1.10) and let $\Omega_{2}$ : $\mathbb{C}^{3} \rightarrow \mathbb{C}$ be an admissible function such that

$$
\operatorname{Re} \Omega_{2}\left(\rho i, \rho i+\frac{\sigma}{k}, \frac{2 k \sigma+\mu}{k(k-1)}+\left(\rho+\frac{v}{k(k-1)}\right) i\right) \leq 0,
$$

where $\rho, \sigma, \eta, v \in \mathbb{R}$,

$$
\sigma \leq-\frac{1}{2}\left(1+\rho^{2}\right), \quad \mu \leq \frac{1}{2}\left(1+\rho^{2}\right) .
$$

If $f \in \mathcal{A}$ satisfies the condition:

$$
\operatorname{Re} \Omega_{2}\left(\frac{B_{k+1}^{c} f(z)}{z}, \frac{B_{k}^{c} f(z)}{z}, \frac{B_{k-1}^{c} f(z)}{z}\right)>0, \quad z \in \mathbb{U},
$$

then

$$
\operatorname{Re}\left(\frac{B_{k+1}^{c} f(z)}{z}\right)>0, \quad z \in \mathbb{U}
$$

Remark 3.1. Our result in Corollary 3.2 is the addition of the results proved in [1].

Taking $(f * g([\alpha+j]))(z)=J_{-a+j, b} f(z)$ and $\lambda_{\alpha+j}=b+1(j=0,1)$, we obtain from (1.7), following recurring relation:

$$
(1+b) J_{-a+j+1, b} f(z)=b J_{-a+j, b} f(z)+z\left(J_{-a+j, b} f(z)\right)^{\prime}(z) .
$$

With the use of recurring relation (3.1), we obtain next consequence of Theorem 2.1 .

Corollary 3.3. Let $J_{-a+j, b} f(z)$ be defined by (1.11) and let $\Sigma: \mathbb{C}^{3} \rightarrow \mathbb{C}$ be an admissible function such that

$$
\operatorname{Re} \Sigma\left(\rho i, \rho i+\frac{\sigma}{b+1}, \frac{2 \sigma}{b+1}+\frac{\sigma+\mu}{(b+1)^{2}}+\left(\rho+\frac{v}{(b+1)^{2}}\right) i\right) \leq 0,
$$

where $\rho, \sigma, \eta, v \in \mathbb{R}$,

$$
\sigma \leq-\frac{1}{2}\left(1+\rho^{2}\right), \quad \mu \leq \frac{1}{2}\left(1+\rho^{2}\right)
$$


If $f \in \mathcal{A}$ satisfies the condition:

$$
\operatorname{Re} \Sigma\left(\frac{J_{-a, b} f(z)}{z}, \frac{J_{-a+1, b} f(z)}{z}, \frac{J_{-a+2, b} f(z)}{z}\right)>0, \quad z \in \mathbb{U},
$$

then

$$
\operatorname{Re}\left(\frac{J_{-a, b} f(z)}{z}\right)>0, \quad z \in \mathbb{U}
$$

Further, taking $(f * h([\alpha-j]))(z)=J_{a-j, b} f(z), \alpha=a$ and $\kappa_{\alpha-j}=1+b, j=0,1$, we obtain, from Theorem 2.2, the next result using recurring relation:

$$
(1+b) J_{a-j-1, b} f(z)=b J_{a-j, b} f(z)+z\left(J_{a-j, b} f(z)\right)^{\prime}(z) .
$$

Corollary 3.4. Let $J_{a-j, b} f(z)$ be defined by (1.11) and let $\Theta: \mathbb{C}^{3} \rightarrow \mathbb{C}$ be an admissible function such that

$$
\operatorname{Re} \Theta\left(\rho i, \rho i+\frac{\sigma}{b+1}, \frac{2 \sigma}{b+1}+\frac{\sigma+\mu}{(b+1)^{2}}+\left(\rho+\frac{v}{(b+1)^{2}}\right) i\right) \leq 0,
$$

where $\rho, \sigma, \eta, v \in \mathbb{R}$,

$$
\sigma \leq-\frac{1}{2}\left(1+\rho^{2}\right), \quad \mu \leq \frac{1}{2}\left(1+\rho^{2}\right)
$$

If $f \in \mathcal{A}$ satisfies the condition:

$$
\operatorname{Re} \Theta\left(\frac{J_{a, b} f(z)}{z}, \frac{J_{a-1, b} f(z)}{z}, \frac{J_{a-2, b} f(z)}{z}\right)>0, \quad z \in \mathbb{U}
$$

then

$$
\operatorname{Re}\left(\frac{J_{a, b} f(z)}{z}\right)>0, \quad z \in \mathbb{U} .
$$

Taking $(f * h([\alpha-j]))(z)=Q_{\beta}^{\alpha-j} f(z)$ and $\kappa_{\alpha-j}=\alpha+\beta-j, j=0,1$, in view of (1.12), we obtain following recurring relation:

$$
(\alpha+\beta) Q_{\beta}^{\alpha-1} f(z)=(\alpha+\beta-1) Q_{\beta}^{\alpha} f(z)+z\left(Q_{\beta}^{\alpha} f(z)\right)^{\prime},
$$

which on applying in Theorem 2.2 gives following result.

Corollary 3.5. Let $Q_{\beta}^{\alpha-j}$ be defined by (1.12) and let $\Xi: \mathbb{C}^{3} \rightarrow \mathbb{C}$ be an admissible function such that

$$
\operatorname{Re} \Xi(A, B, C) \leq 0
$$

where

$$
\begin{gathered}
A=\rho i, \quad B=\rho i+\frac{\sigma}{\alpha+\beta}, \\
C=\left(\frac{1}{\alpha+\beta}+\frac{1}{\alpha+\beta-1}\right) \sigma+\frac{\sigma+\mu}{(\alpha+\beta)(\alpha+\beta-1)}+\left(\rho+\frac{v}{(\alpha+\beta)(\alpha+\beta-1)}\right) i, \\
\rho, \sigma, \eta, v \in \mathbb{R}, \quad \sigma \leq-\frac{1}{2}\left(1+\rho^{2}\right), \quad \mu \leq \frac{1}{2}\left(1+\rho^{2}\right) .
\end{gathered}
$$


If $f \in \mathcal{A}$ satisfies the condition:

$$
\operatorname{Re} \Xi\left(\frac{Q_{\beta}^{\alpha} f(z)}{z}, \frac{Q_{\beta}^{\alpha-1} f(z)}{z}, \frac{Q_{\beta}^{\alpha-2} f(z)}{z}\right)>0, \quad z \in \mathbb{U},
$$

then

$$
\operatorname{Re}\left(\frac{Q_{\beta}^{\alpha} f(z)}{z}\right)>0, \quad z \in \mathbb{U}
$$

Further, taking $(f * g([\alpha+j]))(z)=\Im_{k, \mu}^{m+j} f(z)$ and $\lambda_{\alpha+j}=\frac{\mu+1}{k}, j=0,1$, in view of (1.13), we obtain following recurring relation:

$$
\frac{\mu+1}{k} \Im_{k, \mu}^{m+j+1} f(z)=\left(\frac{\mu+1}{k}-1\right) \Im_{k, \mu}^{m+j} f(z)+z\left(\Im_{k, \mu}^{m+j} f(z)\right)^{\prime}(z) .
$$

With the use of recurring relation (3.2), we obtain next consequence of Theorem 2.1.

Corollary 3.6. Let $\Im_{k, \mu}^{m+j}$ be defined by (1.13) and let $\Sigma_{1}: \mathbb{C}^{3} \rightarrow \mathbb{C}$ be an admissible function such that

$$
\operatorname{Re} \Sigma_{1}\left(\rho i, \rho i+\frac{k}{\mu+1} \sigma, \frac{2 k}{\mu+1} \sigma+\frac{(\sigma+\mu) k^{2}}{(\mu+1)^{2}}+\left(\rho+\frac{\nu k^{2}}{(\mu+1)^{2}}\right) i\right) \leq 0,
$$

where $\rho, \sigma, \eta, v \in \mathbb{R}$,

$$
\sigma \leq-\frac{1}{2}\left(1+\rho^{2}\right), \quad \mu \leq \frac{1}{2}\left(1+\rho^{2}\right)
$$

If $f \in \mathcal{A}$ satisfies the condition:

$$
\operatorname{Re} \Sigma_{1}\left(\frac{\Im_{k, \mu}^{m} f(z)}{z}, \frac{\Im_{k, \mu}^{m+1} f(z)}{z}, \frac{\Im_{k, \mu}^{m+2} f(z)}{z}\right)>0, \quad z \in \mathbb{U},
$$

then

$$
\operatorname{Re}\left(\frac{\Im_{k, \mu}^{m} f(z)}{z}\right)>0, \quad z \in \mathbb{U}
$$

Again, taking $(f * h([\alpha-j]))(z)=\Im_{k, \mu}^{-m-j} f(z)$ and $\kappa_{\alpha-j}=\frac{\mu+1}{k}, j=0,1$, in view of (1.13), we obtain following recurring relation:

$$
\frac{\mu+1}{k} \Im_{k, \mu}^{-m-j-1} f(z)=\left(\frac{\mu+1}{k}-1\right) \Im_{k, \mu}^{-m-j} f(z)+z\left(\Im_{k, \mu}^{-m-j} f(z)\right)^{\prime}(z) .
$$

With the use of recurring relation (3.3), we obtain next consequence of Theorem 2.2.

Corollary 3.7. Let $\Im_{k, \mu}^{-m-j}$ be defined by (1.13) and let $\Theta_{1}: \mathbb{C}^{3} \rightarrow \mathbb{C}$ be an admissible function such that

$$
\operatorname{Re} \Theta_{1}\left(\rho i, \rho i+\frac{k}{\mu+1} \sigma, \frac{2 k}{\mu+1} \sigma+\frac{(\sigma+\mu) k^{2}}{(\mu+1)^{2}}+\left(\rho+\frac{\nu k^{2}}{(\mu+1)^{2}}\right) i\right) \leq 0,
$$

where $\rho, \sigma, \eta, v \in \mathbb{R}$,

$$
\sigma \leq-\frac{1}{2}\left(1+\rho^{2}\right), \quad \mu \leq \frac{1}{2}\left(1+\rho^{2}\right)
$$


If $f \in \mathcal{A}$ satisfies the condition:

$$
\operatorname{Re} \Theta_{1}\left(\frac{\Im_{k, \mu}^{-m} f(z)}{z}, \frac{\Im_{k, \mu}^{-m-1} f(z)}{z}, \frac{\Im_{k, \mu}^{-m-2} f(z)}{z}\right)>0, \quad z \in \mathbb{U},
$$

then

$$
\operatorname{Re}\left(\frac{\Im_{k, \mu}^{-m} f(z)}{z}\right)>0, \quad z \in \mathbb{U} .
$$

Remark 3.2. Taking $(f * g([\alpha]))(z)=\mathbb{D}_{\lambda}^{\nu, n} f(z), \alpha=\nu$ and $\lambda_{\alpha+j}=\nu+1+j, j=0,1$, in Theorem 2.1, we obtain the result proved in [17, Theorem 2.8] which involve a new fractional operator $\mathbb{D}_{\lambda}^{\nu, n}$ defined by (1.14).

Remark 3.3. Similar to the Corollaries 3.1-3.7, we may find the consequent results of Theorem 2.3 and Theorem 2.4 also, on considering certain specific forms of functions $g([\alpha])(z)$ and $h([\alpha])(z)$. We further remark that the results similar to the consequent results obtained in this section, may also be derived for several more general operators defined in the literature which involve some non-zero parameter satisfying a recurring relation. For example, in the recent paper of Srivastava et al. [23], a generalization of Srivastava-Attiya operator is defined which contains, as its special cases, the operators investigated by Prajapat and Bulboacă [11, Eq. (1.8), p. 571$]$, Noor and Bukhari [9, Eq. (1.3), p. 2], Choi et al. [5], Cho and Srivastava [4] etc.

Acknowledgements. The authors wish to thank the reviewers for their valuable suggestions to improve the paper.

\section{REFERENCES}

[1] A. Baricz, E. Deniz, M. Çă̆lar and H. Orhan, Differential subordinations involving generalized Bessel functions, Bull. Malays. Math. Sci. Soc. 38 (2015), 1255-1280.

[2] A. Baricz, Geometric properties of generalized Bessel functions, Publ. Math. Debrecen 73(1-2) (2008), 155-178.

[3] A. Baricz, Geometric properties of generalized Bessel functions of complex order, Mathematica 48(71) (2006), no. 1, 13-18.

[4] N. E. Cho and H. M. Srivastava, Argument estimation of certain analytic functions defined by a class of multiplier transformation, Math. Comput. Modelling 37 (2003), 39-49.

[5] J. H. Choi, M. Saigo and H. M. Srivastava, Some inclusion properties of a certain family of integral operators, J. Math. Anal. Appl. 276 (2002), 432-445.

[6] J. Dziok and H. M. Srivastava, Classes of analytic functions associated with the generalized hypergeometric functions, Appl. Math. Comput. 103 (1999), 1-13.

[7] I. B. Jung, Y. C. Kim and H. M. Srivastava, The Hardy space of analytic functions associated with certain one-parameter families of integral operators, J. Math. Anal. Appl. 176 (1993), 138-147.

[8] S. S. Miller and P. T. Mocau, Second order differential inequalities in the complex plane, J. Math. Anal. Appl. 65 (1978), 289-305.

[9] K. I. Noor and S. Z. H. Bukhari, Some subclasses of analytic and spiral-like functions of complex order involving the Srivastava-Attiya integral operator, Integral Transforms Spec. Funct. 21 (2010), 907-916. 
[10] S. Owa and H. M. Srivastava, Univalent and starlike generalized hypergeometric functions, Canad. J. Math. 39 (1987), 1057-1077.

[11] J. K. Prajapat and T. Bulboacă, Double subordination preserving properties for a new generalized Srivastava-Attiya operator, Chin. Ann. Math. 33 (2012), 569-582.

[12] S. Ponnusamy and M. Vuorinen, Univalence and convexity properties for Gaussian hypergeometric functions, Rocky Mountain J. Math. 31 (2001), 327-353.

[13] R. K. Raina and P. Sharma, Subordination properties of univalent functions involving a new class of operators, Electron. J. Math. Anal. Appl. 2(1) (2014), 37-52.

[14] R. K. Raina and P. Sharma, Subordination preserving properties associated with a class of operators, Le Mathematiche, 68(1) (2013), 217-228.

[15] St. Ruscheweyh, New criteria for univalent functions, Proc. Amer. Math. Soc. 49 (1975), 109-115.

[16] G. S. Sălăgean, Subclass of univalent functions, Complex Analysis: Fifth Romanian Finnish Seminar, Part 1 (Bucharest, 1981), Lecture Notes in Mathematics 1013 (1983), 362-372.

[17] P. Sharma, R. K. Raina and G. S. Sălăgean, Some geometric properties of analytic functions involving a new fractional operator, Mediterr. J. Math. 13(6) (2016), 4591-4605.

[18] P. Sharma, R. K. Raina and J. Sokoł, Certain subordination results involving a class of operators, An. Univ. Oradea Fasc. Mat. 21(2) (2014), 89-99.

[19] P. Sharma and A. K. Bajpai, The convolution of analytic functions involving a parameter, An. Univ. Oradea Fasc. Mat. 23(1) (2016), 87-95.

[20] P. Sharma and A. K. Bajpai, On some analytic functions associated with the linear operators satisfying certain recurring relations, Asian-Eur. J. Math. 9(2) (2016), pp. 13.

[21] H. M. Srivastava and A. A. Attiya, An integral operator associated with the Hurwitz-Lerch zeta function and differential subordination, Integral Transforms Spec. Funct. 18 (2007), 207-216.

[22] H. M. Srivastava and J. Choi, Series Associated with the Zeta and Related Functions, Kluwer Academic Publishers, Dordrecht, Boston, London, 2001.

[23] H. M. Srivastava, S. Gaboury and F. Ghanim, A unified class of analytic functions involving a generalization of the Srivastava-Attiya operator, Appl. Math. Comput. 251 (2015), 35-45.

${ }^{1}$ Department of Mathematics And Astronomy, UNIVERSITY OF LUCKNOW, LUCKNOW 226007 INDIA

E-mail address: sharma_poonam@lkouniv.ac.in

${ }^{2}$ Department of Mathematics and Astronomy, UNIVERSITY OF LUCKNOW, LUCKNOW 226007 INDIA

E-mail address: adityabajpai14@gmail.com 